\title{
Origin of Hopping Conduction in Graphene-Oxide-Doped Silicon Oxide Resistance Random Access Memory Devices
}

\author{
Kuan-Chang Chang, Rui Zhang, Ting-Chang Chang, Senior Member, IEEE, Tsung-Ming Tsai, J. C. Lou, \\ Jung-Hui Chen, Tai-Fa Young, Min-Chen Chen, Ya-Liang Yang, Yin-Chih Pan, Geng-Wei Chang, \\ Tian-Jian Chu, Chih-Cheng Shih, Jian-Yu Chen, Chih-Hung Pan, Yu-Ting Su, Yong-En Syu, \\ Ya-Hsiang Tai, and Simon M. Sze, Life Fellow, IEEE
}

\begin{abstract}
In this letter, a double-active-layer ( $\mathrm{Zr}: \mathrm{SiO}_{x} /$ $\left.\mathrm{C}: \mathrm{SiO}_{x}\right)$ resistive switching memory device with a high ON/OFF resistance ratio and small working current $(0.02 \mathrm{~mA})$, is presented. Through the analysis of Raman and Fourier transform infrared spectroscopy spectra, we find that graphene oxide exists in the $\mathrm{C}: \mathrm{SiO}_{x}$ layer. It can be observed that $\mathrm{Zr}: \mathrm{SiO}_{x} / \mathrm{C}: \mathrm{SiO}_{x}$ structure has superior switching performance and higher stability compared with the single-active-layer $\left(\mathrm{Zr}: \mathrm{SiO}_{x}\right)$ structure, which is attributed to the existence of graphene oxide flakes formed during the sputter process. $I-V$ characteristics under a series of increasing temperature were analyzed to testify the carrier hopping distance variation, which is further verified by our graphene oxide redox reaction model.
\end{abstract}

Index Terms - conduction, graphene oxide, hopping, redox reaction, resistance random access memory (RRAM).

\section{INTRODUCTION}

$\mathbf{T}$ O SURMOUNT the technical and physical limitation issues of conventional charge-storage-based memories

Manuscript received December 19, 2012; revised February 20, 2013; accepted February 26, 2013. Date of publication March 27, 2013; date of current version April 22, 2013. This work was supported by the National Science Council of the Republic of China under Contract NSC-101-2120-M110-002, and Contract NSC 101-2221-E-110-044-MY3. The review of this letter was arranged by Editor T. San.

K. C. Chang, T. M. Tsai, Y.-C. Pan, T.-J. Chu, and C.-H. Pan are with the Department of Materials and Optoelectronic Science, National Sun Yat-Sen University, Kaohsiung 80424, Taiwan.

R. Zhang and J. C. Lou are with the School of Software and Microelectronics, Peking University, Beijing 100871, China.

T.-C. Chang, M.-C. Chen, J.-Y. Chen, Y.-T. Su, and Y.-E. Syu are with the Department of Physics, National Sun Yat-Sen University, Kaohsiung 80424, Taiwan, and also with the Advanced Optoelectronics Technology Center, National Cheng Kung University, Taiwan (e-mail: tcchang@mail.phys.nsysu.edu.tw).

J.-H. Chen and C.-C. Shih are with the Department of Chemistry, National Kaohsiung Normal University, Kaohsiung, Taiwan.

T.-F. Young and Y.-L. Yang are with the Department of Mechanical \& Electro-Mechanical Engineering, National Sun Yat-Sen University, Kaohsiung 80424, Taiwan.

G. W. Chang and Y. H. Tai are with the Department of Photonics and the Institute of Electro-Optical Engineering, National Chiao Tung University, Hsinchu 300, Taiwan.

S. M. Sze is with the Department of Physics, National Sun Yat-Sen University, Kaohsiung 80424, Taiwan, and also with the Department of Electronics Engineering, National Chiao Tung University, Hsinchu 300, Taiwan.

Color versions of one or more of the figures in this letter are available online at http://ieeexplore.ieee.org.

Digital Object Identifier 10.1109/LED.2013.2250899
[1]-[6], the resistance random access memory (RRAM) is constructed of an insulating layer sandwiched by two electrodes. This structure is a great potential candidate for next-generation nonvolatile memory due to its superior characteristics such as lesser cost, simple structure, high-speed operation, and nondestructive readout [7]-[16].

Recent years have witnessed many breakthroughs in the research on graphene as well as a significant advance in the mass production of this material. This one-atom-thick fabric has many supreme properties, like extreme mechanical strength and high electronic and thermal conductivities.

In our research, double resistive switching layers with a sandwiched structure of $\mathrm{Pt} / \mathrm{Zr}: \mathrm{SiO}_{x} / \mathrm{C}: \mathrm{SiO}_{x} / \mathrm{TiN}$ were fabricated. Meanwhile, single-resistive-switching-layer RRAM $\left(\mathrm{Pt} / \mathrm{Zr}: \mathrm{SiO}_{x} / \mathrm{TiN}\right)$ was also fabricated so as to make a comparison. From the analysis of Raman and Fourier transform infrared spectroscopy (FTIR) spectra, graphene oxide was found, and that was the reason why $\mathrm{Zr}: \mathrm{SiO}_{x} / \mathrm{C}: \mathrm{SiO}_{x}$ structure showed better performance of resistive switching characteristics and had lower forming voltage.

As hopping conduction mechanism was found in current fitting of $\mathrm{Zr}: \mathrm{SiO}_{x} / \mathrm{C}: \mathrm{SiO}_{x}$ RRAM, $I-V$ measurement under a series of increasing temperature was performed to further obtain carrier hopping distance. Meanwhile, the hopping distance variation was demonstrated by our graphene oxide redox reaction model.

\section{EXPERIMENTAL SETUP}

The experimental specimens were prepared as follows. For the single-active-layer specimen, the $\mathrm{Zr}: \mathrm{SiO}_{x}$ thin film (around $20 \mathrm{~nm}$ ) was deposited on the $\mathrm{TiN} / \mathrm{Ti} / \mathrm{SiO}_{2} / \mathrm{Si}$ substrate by cosputtering with the pure $\mathrm{SiO}_{2}$ and $\mathrm{Zr}$ targets. However, for the double-resistive-switching-layer specimen, first a $\mathrm{C}: \mathrm{SiO}_{x}$ film (around $6 \mathrm{~nm}$ ) was deposited by cosputtering with the $\mathrm{SiO}_{2}$ and $C$ targets. Then the layer of $\mathrm{Zr}: \mathrm{SiO}_{x}$ (around $14 \mathrm{~nm}$ ) was deposited with the same RF power, argon ambient and working pressure as the antecedent single- $\mathrm{Zr}: \mathrm{SiO}_{x}$-layer specimen

Ultimately, the Pt top electrode of $200 \mathrm{~nm}$ thickness was deposited on both specimens by DC magnetron sputtering. 


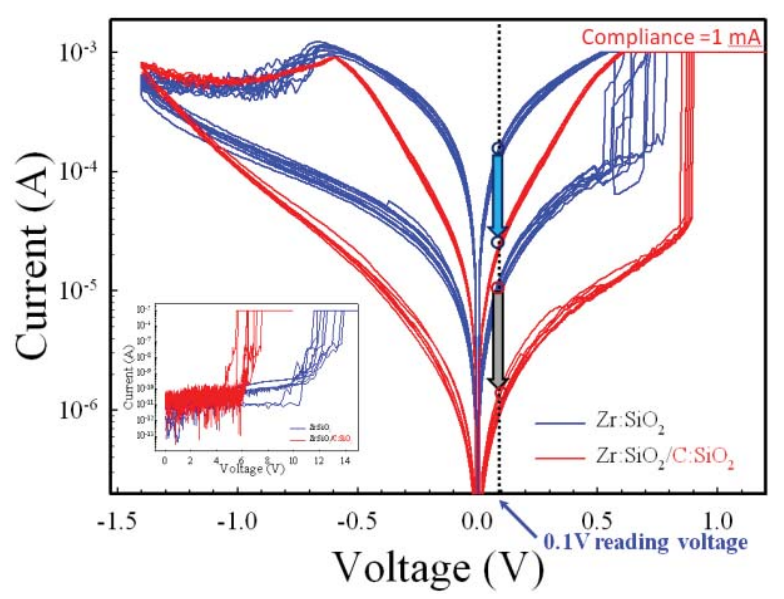

Fig. 1. Blue and red curves are the resistive switching characteristics of $\mathrm{Zr}: \mathrm{SiO}_{x}$ film and $\mathrm{Zr}: \mathrm{SiO}_{x} / \mathrm{C}: \mathrm{SiO}_{x}$ film, respectively. The current of $\mathrm{Zr}: \mathrm{SiO}_{x} /$ $\mathrm{C}: \mathrm{SiO}_{x}$ film is reduced on both high resistance state (HRS) and low resistance state (LRS). The figure on bottom left is a forming curve, which shows that $\mathrm{Zr}: \mathrm{SiO}_{x} / \mathrm{C}: \mathrm{SiO}_{x}$ film has smaller forming voltage.

The entire electrical measurements of devices with the $\mathrm{Pt}$ electrode of $250-\mu \mathrm{m}$ diameter were performed using Agilent B1500 semiconductor parameter analyzer. Besides, FTIR and Raman spectroscopy were used to analyze the chemical composition and bonding of these insulator materials, respectively.

\section{RESULTS AND DISCUSSION}

The "forming process" is required to activate all of the RRAM devices, using DC voltage sweeping with a compliance current of $10 \mu \mathrm{A}$. Compared with $\mathrm{Zr}: \mathrm{SiO}_{x} \mathrm{RRAM}$, $\mathrm{Zr}: \mathrm{SiO}_{x} / \mathrm{C}: \mathrm{SiO}_{x} \mathrm{RRAM}$ devices have smaller forming voltage around 5-7 V (inset of Fig. 1), and the working current of the $\mathrm{Zr}: \mathrm{SiO}_{x} / \mathrm{C}: \mathrm{SiO}_{x} \mathrm{RRAM}$ devices in both low-resistance state (LRS) and high-resistance state (HRS) is lower than that of the $\mathrm{Zr}: \mathrm{SiO}_{x}$ devices (Fig. 1).

To investigate the interesting phenomena, we utilized the material spectra analyses to find out the reason of working current reduction and better stability. The $\mathrm{C}: \mathrm{SiO}_{x}$ film was analyzed by Raman spectroscopy, from which we found typical graphene oxide Raman spectra which comprised of a higher $G$ band peak and a lower $D$ band peak (Fig. 2) [17]. In order to further testify the existence of graphene oxide and find its chemical bonding type, FTIR was used to analyze the $\mathrm{C}: \mathrm{SiO}_{x}$ film. Graphene-oxide-coupled $\mathrm{OH}$ peak can be observed at the wave number of $3665 \mathrm{~cm}^{-1}$, as shown in the top right FTIR spectra of Fig. 2.

Through current fitting, we found that single-resistiveswitching-layer devices exhibit Poole-Frenkel conduction in HRS and Ohmic conduction in LRS, while both LRS and HRS are hopping conduction mechanisms for double-active-layer devices. Then, we carried out $I-V$ measurement under a series of increasing temperature, which is shown in Fig. 3(a) for ON state and in Fig. 3(c) for OFF state. According to the relationship of hopping conduction, $J=q N a v_{0} e^{-q \phi_{T} / k T} e^{q a V / 2 d k T}$, where $N, a, \phi_{T}, v_{0}$, and $d$ are density of space charge, mean of hopping distance, barrier height of hopping, intrinsic vibration frequency, and film thickness, respectively, we can draw out

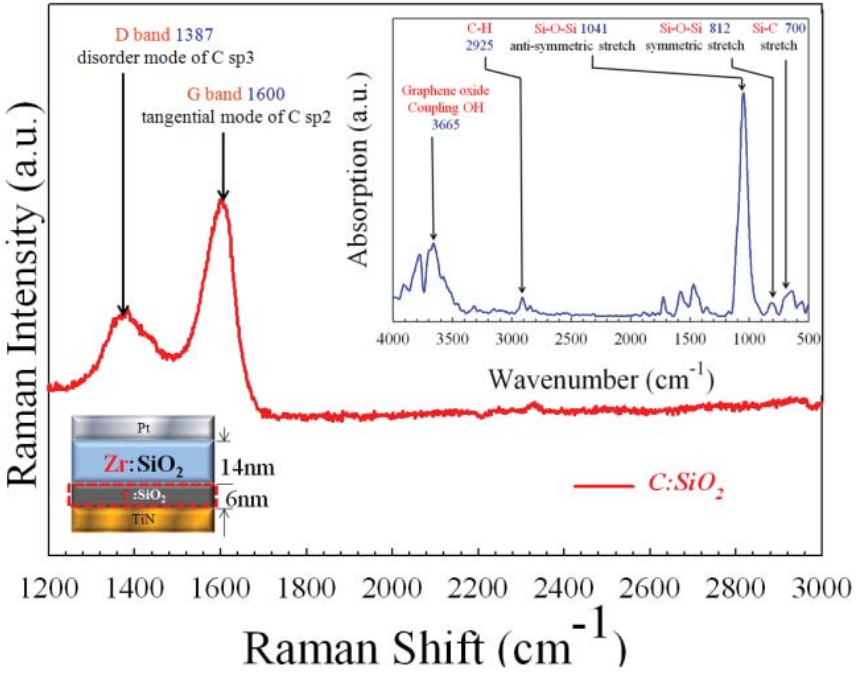

Fig. 2. Raman spectra of $C$ sp2 and $C$ sp3 in $\mathrm{C}: \mathrm{SiO}_{x}$ film. It confirms the existence of grapheme oxide. The blue curve on upper right is the corresponding FTIR spectra from which grapheme oxide coupling $\mathrm{OH}$ peak can be observed at the wavenumber of $3665 \mathrm{~cm}^{-1}$.
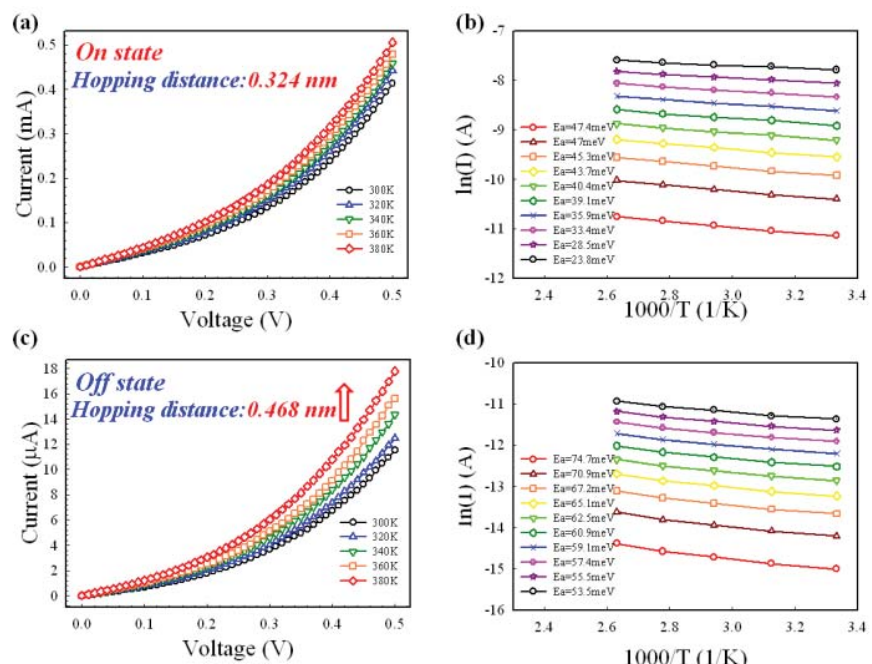

(d)

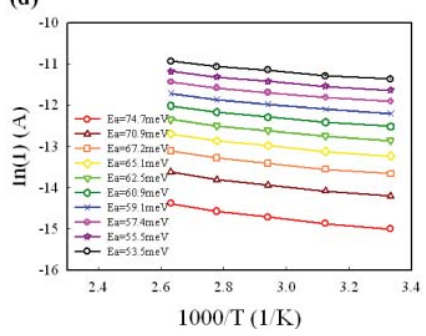

Fig. 3. (a) and (c) ON and OFF state current-voltage characteristics of $\mathrm{Zr}: \mathrm{SiO}_{x} / \mathrm{C}: \mathrm{SiO}_{x} \mathrm{RRAM}$ under increasing temperature. The calculated hopping distance is 0.32 and $0.46 \mathrm{~nm}$, respectively. (b) and (d) Corresponding ON and OFF state activation energy under different voltage.

the curve $\phi_{T}-a V / 2 d$ with a vertical axis of $\operatorname{In}(I)$ and a lateral axis of 1000/T [Fig. 3(b) for ON state and Fig. 3(d) for OFF state]. As $d$ stands for hopping distance, we calculated from the curves in Fig. 3(b) and (d) the corresponding hopping distance for ON state and OFF state as 0.324 and $0.468 \mathrm{~nm}$, respectively. The result showed an increase of $0.144 \mathrm{~nm}$ in the hopping distance in HRS as compared with LRS.

On the basis of the electrical and material analyses, we proposed a reaction model to explain the transfer of carrier conduction mechanism of the $\mathrm{Zr}: \mathrm{SiO}_{x} / \mathrm{C}: \mathrm{SiO}_{x}$ film as shown in Fig. 4. The conductive filament will be formed in the $\mathrm{Zr}: \mathrm{SiO}_{x}$ film after the forming process and it will be connected with graphene oxide flake in the switching region of $\mathrm{C}: \mathrm{SiO}_{x}$ film. Meanwhile, oxygen-contained groups are removed from graphene oxide and are driven to the direction of the bottom electrode. The carrier will hop through carbon atoms within 


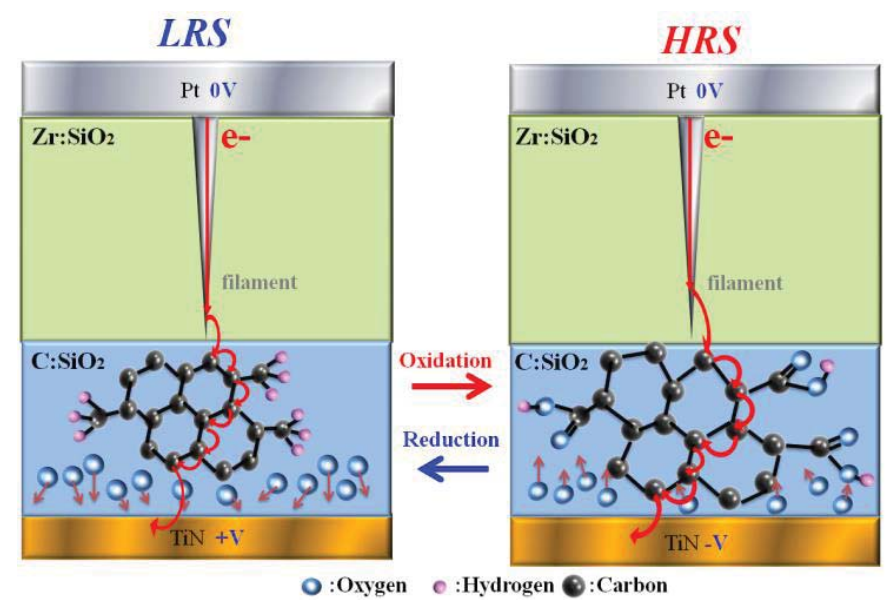

Fig. 4. Schematic diagram of graphene oxide redox reaction in $\mathrm{Zr}: \mathrm{SiO}_{x} /$ $\mathrm{C}: \mathrm{SiO}_{x}$ RRAM. The hopping distance variation is due to the stretching of carbon-carbon bond within the carbocycle.

the carbocycle in the $\mathrm{C}: \mathrm{SiO}_{x}$ film. If the bottom $\mathrm{TiN}$ electrode is applied with a negative bias to perform a reset process, oxygen atoms are repelled to the reverse direction of TiN electrode and are absorbed by graphene oxide. With the absorption of oxygen atoms, carbon-carbon bonds are stretched and carbocycle is enlarged, which results in longer hopping distance of carriers In single-layer RRAM devices, in LRS, electrons conduct through metal filaments from top electrode to bottom electrode, and in HRS, electrons conduct through shallow defects between the tip of the ruptured filament and the bottom TiN electrode. But in double-layer devices, the oxygencontained groups' absorption and desorption of graphene oxide result in carbocycle deformation and thus the hopping distance variation between $\mathrm{ON}$ and OFF states in graphene-oxide-doped silicon RRAM.

\section{CONCLUSION}

By cosputtering $C$ and $\mathrm{Zr}$ with $\mathrm{SiO}_{2}$ we fabricated a double-resistive-switching-layer RRAM that has obvious lower working current and forming voltage. Both FTIR and Raman spectra confirmed the existence of graphene oxide. With the absorption and desorption of oxygen atoms, carbon-carbon bonds within carbocycle are stretched, which results in hopping distance variation and resistive switch. The $\mathrm{Zr}: \mathrm{SiO}_{x} / \mathrm{C}: \mathrm{SiO}_{x}$ structure has superior switching performance and higher stability compared with the single-active-layer $\left(\mathrm{Zr}: \mathrm{SiO}_{x}\right)$ structure, which is attributed to the redox reaction of graphene oxide.

\section{ACKNOWLEDGMENT}

This work was performed at the National Science Council Core Facilities Laboratory for Nano-Science and NanoTechnology in the Kaohsiung-Pingtung area.

\section{REFERENCES}

[1] J. Liu, Q. Wang, S. B. Long, M. H. Zhang, and M. Liu, "A metal/ $/ \mathrm{Al}_{2} \mathrm{O}_{3} / \mathrm{ZrO}_{2} / \mathrm{SiO}_{2} / \mathrm{Si}$ (MAZOS) structure for high-performance non-volatile memory application," Semicond. Sci. Technol., vol. 25, no. 5 , p. 055013 , May 2010.
[2] D. D. Jiang, M. H. Zhang, Z. L. Huo, Q. Wang, J. Liu, Z. A Yu, X. N. Yang, Y. Wang, B. Zhang, J. N. Chen, and M. Liu, "A study of cycling induced degradation mechanisms in Si nanocrystal memory devices," Nanotechnology, vol. 22, no. 25, p. 254009, Jun. 2011.

[3] F. M. Yang, T. C. Chang, P. T. Liu, P. H. Yeh, Y. C. Yu, J. Y. Lin, S. M. Sze, and J. C. Lou, "Nickel nanocrystals with $\mathrm{HfO}_{2}$ blocking oxide for nonvolatile memory application," Appl. Phys. Lett., vol. 90, no. 22, pp. 222104-1-222104-3, May 2007.

[4] T. C. Chang, F. Y. Jian, S. C. Chen, and Y. T. Tsai, "Developments in nanocrystal memory," Mater. Today, vol. 14, no. 12, pp. 608-615, Dec. 2011.

[5] F. M. Yang, T. C. Chang, P. T. Liu, U. S. Chen, P. H. Yeh, Y. C. Yu, J. Y. Lin, S. M. Sze, and J. C. Lou, "Nickel nanocrystals with $\mathrm{HfO}_{2}$ blocking oxide for nonvolatile memory application," Appl. Phys. Lett., vol. 90, no. 22, p. 222104, May 2007.

[6] C. X. Zhu, Z. L. Huo, Z. G. Xu, M. H. Zhang, Q. Wang, J. Liu, S. Long, and M. Liu, "Performance enhancement of multi-level cell nonvolatile memory by using a bandgap engineered high- $\kappa$ trapping layer," Appl. Phys. Lett., vol. 97, no. 25, pp. 253503-1-253503-3, 2010.

[7] Y. Wang, Q. Liu, S. B. Long, W. Wang, Q. Wang, M. H. Zhang, S. Zhang, Y. T. Li, Q. Y. Zuo, J. H. Yang, and M. Liu, "Investigation of resistive switching in $\mathrm{Cu}$-doped $\mathrm{HfO}_{2}$ thin film for multilevel non-volatile memory applications," Nanotechnology, vol. 21, no. 4, p. 045202, Jan. 2010.

[8] Y. E. Syu, T. C. Chang, T. M. Tsai, Y. C. Hung, K. C. Chang, M. J. Tsai, M. J. Kao, and S. M. Sze, "Redox reaction switching mechanism in RRAM device with $\mathrm{Pt} / \mathrm{CoSiO}_{x} / \mathrm{TiN}$ structure," IEEE Electron Device Lett., vol. 32, no. 4, pp. 545-547, Apr. 2011

[9] K. C. Chang, T. M. Tsai, T. C. Chang, Y. E. Syu, C. C. Wang, S. L. Chuang, C. H. Li, D. S. Gan, and S. M. Sze, "Reducing operation current of Ni-doped silicon oxide resistance random access memory by supercritical $\mathrm{CO}_{2}$ fluid treatment," Appl. Phys. Lett., vol. 99, no. 26 , pp. 263501-1-263501-4, Dec. 2011.

[10] K. C. Chang, T. M. Tsai, T. C. Chang, Y. E. Syu, K. H. Liao, S. L. Chuang, C. H. Li, D. S. Gan, and S. M. Sze, "The effect of silicon oxide based RRAM with tin doping," Electrochem. Solid-State Lett., vol. 15, no. 3, pp. H65-H68, 2012.

[11] T. M. Tsai, K. C. Chang, T. C. Chang, Y. E. Syu, K. H. Liao, B. H. Tseng, and S. M. Sze, "Dehydroxyl effect of Sn-doped silicon oxide resistance random access memory with supercritical $\mathrm{CO}_{2}$ fluid treatment," Appl. Phys. Lett., vol. 101, no. 11, pp. 112906-1-112906-4, Sep. 2012.

[12] T. M. Tsai, K. C. Chang, T. C. Chang, Y. E. Syu, S. L. Chuang, S. Lan, C. G. Wei, L. G. Ru, C. M. Chen, H. H. Chun, L. S. Kun, T. Y. Hsiang, G. D. Shin, Y. Y. Liang, Y. T. Fa, T. B. Heng, C. K. Huang, T. M. Jinn, C. Y. H. Wang, and S. M. Sze, "Bipolar resistive RAM characteristics induced by nickel incorporated into silicon oxide dielectrics for IC applications," IEEE Electron Device Lett., vol. 33, no. 12, pp. 1696-1698, Dec. 2012.

[13] T. M. Tsai, K. C. Chang, T. C. Chang, G. W. Chang, Y. E. Syu, Y. En, S. Y. Ting, L. G. Ru, L. K. Hsiao, C. M. Chen, H. H. Chun, T. Y. Hsiang, G. D. Shin, Y. Cong, W. Hao, and S. M. Sze, "Origin of hopping conduction in sn-doped silicon oxide RRAM with supercritical $\mathrm{CO}_{2}$ fluid treatment," IEEE Electron Device Lett., vol. 33, no. 12, pp. 1693-1695, Dec. 2012.

[14] Y. T. Li, S. B. Long, M. H. Zhang, Q. Liu, S. Zhang, H. Manhong, Q. Liu, L. Shao, S. Zhang, Y. Y. Wang, Q. Zuo, S. Liu, and M. Liu, "Resistive switching properties of $\mathrm{Au} / \mathrm{ZrO}_{2} / \mathrm{Ag}$ structure for low voltage nonvolatile memory applications," IEEE Electron Device Lett., vol. 31, no. 2, pp. 117-119, Feb. 2010.

[15] J. Yao, Z. Sun, L. Zhong, D. Natelson, and J. M. Tour, "Resistive switches and memories from silicon oxide," Nano Lett., vol. 10, no. 10 , pp. $4105-4110,2010$

[16] A. Mehonic, S. Cueff, M. Wojdak, S. Hudziak, O. Jambois, C. Labbé, B. Garrido, R. Rizk, and A. J. Kenyon, "Resistive switching in silicon suboxide films," J. Appl. Phys., vol. 111, no. 7, pp. 074507-1-074507-9, Apr. 2012.

[17] N. Hu, Y. Wang, J. Chai, R. Gao, Z. Yang, E. S. W. Kong, and Y. Zhang, "Gas sensor based on p-phenylenediamine reduced graphene oxide," Sensors Actuat. B. Chem., vol. 163, no. 1, pp. 107-114, Mar. 2012. 